\title{
RESEARCH IN BASIC SCIENCE
}

\section{Effect of High-Dose Sodium Bicarbonate on the Vasopressor Effects of Epinephrine During Cardiopulmonary Resuscitation}

\author{
Barry E. Bleske, Pharm.D., Eric W. Warren, Pharm.D., Ted L. Rice, M.S., Lori J. Gilligan, L.V.T., and \\ Alan R. Tait, Ph.D.
}

We attempted to determine the effect of extreme alkalemia induced by highdose sodium bicarbonate on the vasopressor effects of epinephrine during cardiopulmonary resuscitation (CPR). Subjects in this randomized, blinded study performed in a controlled laboratory environment were 12 mongrel dogs that had had a previous episode of CPR. Each dog underwent 3 minutes of ventricular fibrillation (VF) followed by 7 minutes of closed-chest CPR. Animals were assigned to receive either sodium bicarbonate $3 \mathrm{mEq} / \mathrm{kg}$ and epinephrine $0.1 \mathrm{mg} / \mathrm{kg}$, or normal saline $3 \mathrm{ml} / \mathrm{kg}$ and epinephrine $0.1 \mathrm{mg} / \mathrm{kg}$. The sodium bicarbonate or normal saline was infused over 2 minutes beginning at 4 minutes of VF ( $1 \mathrm{~min}$ of CPR) followed by bolus epinephrine. Arterial $\mathrm{pH}$ in the sodium bicarbonate group was significantly higher at each sampling point $(7.7 \pm 0.1$ vs $7.29 \pm 0.06$ at 1 min after drug, $p<0.001)$. However, there were no statistically or clinically significant differences in coronary perfusion pressure between the groups at any time: $29 \pm 13$ versus $32 \pm 21 \mathrm{~mm} \mathrm{Hg} 1$ minute, and $22 \pm 12$ versus $26 \pm 19 \mathrm{~mm} \mathrm{Hg} 4$ minutes after epinephrine for sodium bicarbonate and normal saline, respectively $(\mathrm{p}>0.7)$. Increased arterial $\mathrm{pH}$ (alkalemia) induced by high-dose sodium bicarbonate administration did not improve the vasopressor effects of epinephrine during $C P R$ in this canine model. These results suggest the limited value of administering sodium bicarbonate during CPR to improve the responsiveness to epinephrine.

(Pharmacotherapy 1995;15(5):660-664)

During cardiopulmonary resuscitation (CPR), the development of systemic acidosis and acidemia may alter the efficacy of adrenergic agents. ${ }^{1-4}$ The role of these drugs, especially epinephrine, during $\mathrm{CPR}$ is to increase coronary

From the University of Michigan, College of Pharmacy (Drs. Bleske and Warren, and Mr. Rice), and the Departments of Pharmacy (Drs. Bleske and Warren, and Mr. Rice) and Anesthesiology (Ms. Gilligan and Dr. Tait), University of Michigan Hospitals, Ann Arbor, Michigan.

Address reprint requests to Barry E. Bleske, Pharm.D., University of Michigan, College of Pharmacy, Ann Arbor, MI 48109-1065. perfusion pressure and help improve the chances for successful resuscitation. Alterations in the vasopressor properties of epinephrine may have important consequences in this regard.

Theoretically, the treatment of acidemia may improve the vasopressor response to epinephrine, since acidotic conditions decrease the vasopressor effects of catecholamines. However, a previous study showed that a standard dose of sodium bicarbonate $(1 \mathrm{mEq} / \mathrm{kg})$ does not alter the vasopressor effect of epinephrine or improve the resuscitation rate. ${ }^{5}$ That trial only partly 
evaluated the effect of sodium bicarbonate on the vasopressor effects of epinephrine. What was not determined was the effect of extreme elevations of $\mathrm{pH}$ during $\mathrm{CPR}$, which may occur especially after administration of large doses of sodium bicarbonate. In addition, if any alterations in epinephrine's effect occur, it should be apparent with extreme changes in $\mathrm{pH}$. Therefore, we assessed the effect of severe alkalemia induced by sodium bicarbonate on the vasopressor effects of epinephrine during CPR in a canine model.

\section{Methods}

Animal Preparation

In this blinded trial, 12 adult mongrel dogs $(20.1 \pm 3.2 \mathrm{~kg})$ that had been resuscitated from an episode of cardiac arrest with CPR were randomized to one of two treatment groups by a random numbers table. All experiments were of similar design, involving epinephrine administration and duration ( $10 \mathrm{~min}$ of ventricular fibrillation). Animal care and procedures were in accord with national and institutional guidelines and approved by the Unit for Laboratory Medicine.

Animals were initially anesthetized with pentobarbital $25 \mathrm{mg} / \mathrm{kg}$ intravenous injection. Anesthesia was maintained with additional doses of pentobarbital $5 \mathrm{mg} / \mathrm{kg}$ as required. All animals were stabilized for at least 30 minutes after supplemental doses of pentobarbital before beginning the experimental procedure. Each animal was secured in a supine position on a surgical table with a thermoblanket to maintain a body temperature of $37^{\circ} \mathrm{C}$. A cuffed endotracheal tube was placed, and ventilations controlled with an Anesthesia Ventilator (Ohio Medical Products, Madison, WI), with a fraction of inspired oxygen of 1.0. Initially, the tidal volume was set at 10-15 $\mathrm{ml} / \mathrm{kg}$ with a respiratory rate of 12 breaths/ minute. Tidal volume and respiratory rate were adjusted as necessary to achieve and maintain an arterial $\mathrm{pH}$ of $7.40 \pm 0.10$ and a partial pressure of carbon dioxide $\left(\mathrm{pCO}_{2}\right)$ of $40 \pm 10 \mathrm{~mm} \mathrm{Hg}$. Arterial blood samples were collected in heparinized syringes, capped, placed in ice, and analyzed using a Radiometer ABL2 (Acid-Base Laboratory, Cleveland, $\mathrm{OH}$ ) within 5 minutes of sampling.

After surgical exposure of a femoral artery and vein and jugular vein, catheters were placed. Specifically, a $6 \mathrm{~F}$ pigtailed catheter was placed in the aorta for pressure measurements, epinephrine administration, and arterial blood gas sampling.
Additional $6 \mathrm{~F}$ catheters were placed in the right atrium to measure pressure, and in the right ventricle to induce ventricular fibrillation (VF) and administer sodium bicarbonate. All pressures were measured with a Gould P23 pressure transducer (after calibration) and recorded together with a single-lead electrocardiogram by a Gould 8-channel RS 3800 graphic recorder (Gould Inc., Cleveland, $\mathrm{OH}$ ). Catheter position was determined by evaluating the pressure waveforms and was confirmed by autopsy if necessary. After catheter placement, intravenous heparin $150 \mathrm{U} / \mathrm{kg}$ was administered to help ensure catheter patency.

\section{Experimental Protocol}

After obtaining baseline measurements, VF was induced by placing a pacing wire in the right ventricle and applying a $24-\mathrm{mA}, 60-\mathrm{Hz}$ electrical current to the ventricle wall. After 3 minutes of unassisted ventricular fibrillation (without ventilation), CPR was begun using a pneumatic chest-compression device (Thumper; Michigan Instruments, Grand Rapids, MI) set at a compression rate of $80 /$ minute with a compression duration of 0.5 seconds. Initially, the compression force was set to produce a stable coronary perfusion pressure gradient (aortic-right atrial middiastolic pressure) below $30 \mathrm{~mm} \mathrm{Hg}$. After every fifth compression, diastole was prolonged by 0.5 seconds, and the lungs were inflated with an inspiratory pressure setting of approximately $15 \mathrm{~cm} \mathrm{H}_{2} \mathrm{O}$ with $100 \%$ oxygen.

After 1 minute of CPR ( 4 min VF), animals were randomized to receive either sodium bicarbonate $1 \mathrm{mEq} / \mathrm{mL}$ solution $(3 \mathrm{mEq} / \mathrm{kg}$ ) or normal saline $(3 \mathrm{ml} / \mathrm{kg})$ administered into the right ventricle over a 2 -minute period, followed by intravenous epinephrine $0.1 \mathrm{mg} / \mathrm{kg}$ bolus administered into the aorta, followed by a $10-\mathrm{ml}$ saline flush ( $6 \mathrm{~min} V F)$. Pressure measurements and blood gas samples were obtained every minute after the end of the sodium bicarbonate or normal saline infusion until termination of the study (10 min VF).

\section{Data Analysis}

Pressures were determined using an average of five readings of the pressure waveforms per pressure measurement by a blinded investigator. Statistical comparison of the treatment groups was performed by analysis of variance with a repeated measures design, and Student's $t$ test with Bonferroni's correction where appropriate. 
Table 1. Arterial Blood Gas Values $(n=6)$

\begin{tabular}{|c|c|c|c|c|c|}
\hline & Baseline & $1^{a}$ & 2 & 3 & 4 \\
\hline $\begin{array}{l}\text { Bicarbonate } \\
\text { pH } \\
\mathrm{pCO}_{2}(\mathrm{~mm} \mathrm{Hg}) \\
\mathrm{HCO}_{3}^{-}(\mathrm{mEq} / \mathrm{L})\end{array}$ & $\begin{aligned} 7.32 & \pm 0.04 \\
40 & \pm 4.1 \\
20.2 & \pm 2.0\end{aligned}$ & $\begin{array}{l}7.70 \pm 0.1^{b} \\
48.8 \pm 8.2 \\
61.0 \pm 11.2^{b}\end{array}$ & $\begin{array}{l}7.65 \pm 0.2^{\mathrm{b}} \\
41.2 \pm 6.8 \\
48.1 \pm 21^{\mathrm{b}}\end{array}$ & $\begin{array}{l}7.59 \pm 0.19^{b} \\
35.8 \pm 8.0 \\
34.9 \pm 10.4^{b}\end{array}$ & $\begin{array}{l}7.55 \pm 0.22^{\mathrm{b}} \\
34.5 \pm 7.8 \\
30.8 \pm 10.3^{\mathrm{b}}\end{array}$ \\
\hline $\begin{array}{l}\text { Normal saline } \\
\mathrm{pH} \\
\mathrm{pCO}_{2}(\mathrm{~mm} \mathrm{Hg}) \\
\mathrm{HCO}_{3}^{-}(\mathrm{mEq} / \mathrm{L})\end{array}$ & $\begin{array}{c}7.33 \pm 0.04 \\
41 \pm 5.5 \\
21 \pm 2.4\end{array}$ & $\begin{array}{l}7.29 \pm 0.06 \\
34.5 \pm 14.7 \\
15.5 \pm 5.3\end{array}$ & $\begin{array}{l}7.28 \pm 0.07 \\
34.4 \pm 13.9 \\
14.9 \pm 4.9\end{array}$ & $\begin{array}{l}7.25 \pm 0.08 \\
32.3 \pm 13.0 \\
13.2 \pm 4.2\end{array}$ & $\begin{array}{l}7.26 \pm 0.07 \\
33.2 \pm 13.8 \\
14.2 \pm 5.0\end{array}$ \\
\hline
\end{tabular}

Data are reported as mean and standard deviation.

\section{Results}

All 12 animals completed the protocol and were included in the data analysis. Arterial blood gas values for each treatment group throughout the study period are shown in Table 1 . No significant differences were observed at baseline. As expected, however, significant differences in $\mathrm{pH}$ and bicarbonate concentration were present after administration of sodium bicarbonate compared with normal saline. All animals in the sodium bicarbonate group had $\mathrm{pH}$ values above 7.5 , indicating arterial alkalemia. Three of these animals had extremely elevated $\mathrm{pH}$ values, above 7.75. In the normal saline group, all animals had a $\mathrm{pH}$ of 7.4 or below. In four of these animals the values were 7.3 or below at the time of peak pressure change after epinephrine administration.

Baseline blood pressures were measured to ensure that both groups were comparable. This was important, since each animal had experienced a previous episode of VF and CPR of similar duration. No significant differences were seen in baseline aortic systolic or diastolic pressures between the two groups: sodium bicarbonate $145 \pm 45$ and $93 \pm 40 \mathrm{~mm} \mathrm{Hg}$; normal saline $133 \pm 37$ and $88 \pm 30 \mathrm{~mm} \mathrm{Hg}$ (p>0.6).

No significant difference was seen between the groups with regard to absolute pressure changes (aortic systolic, aortic diastolic, right atrial diastolic, coronary perfusion pressure) at any time point measured (Table 2 ). In addition, when the data were analyzed by percentage change from baseline, there was no significant difference between the groups. As shown in Figure 1, the two groups, with the exception of one animal, appear visually to be identical with regard to peak change in coronary perfusion pressure. In support of this, no statistical difference occurred in the mean change from baseline to peak pressure between the two groups $(12.5 \pm 8.9$ and $20.1 \pm 120 \mathrm{~mm} \mathrm{Hg}$ sodium bicarbonate and normal saline, respectively; $p>0.05)$. When the one apparent outlier in the normal saline group was excluded, the mean peak change in pressure between the two groups were identical $(12.5 \pm 8.9$ and $12.7 \pm 8.9 \mathrm{~mm} \mathrm{Hg}$ sodium bicarbonate and normal saline, respectively; $p>0.05$ ).

\section{Discussion}

Significant arterial alkalemia was induced by the administration of sodium bicarbonate compared with normal saline. As expected, dogs in the normal saline group developed arterial acidemia during CPR. Despite the difference in arterial blood gas values, no significant difference was seen in the vasopressor response to epinephrine between the groups.

Studies have shown that the vasopressor effect of catecholamines may be decreased in the presence of acidosis. ${ }^{1-4}$ Theoretically therefore, the treatment of acidosis may improve the response to catecholamines. This may have important implications especially during CPR, when systemic acidosis and arterial and venous acidemia occur, and when acidosis is treated by ventilation and sodium bicarbonate administration.

In our earlier study, standard-dose sodium bicarbonate $1 \mathrm{mEq} / \mathrm{kg}$ did not improve the response to epinephrine during $\mathrm{CPR},{ }^{5}$ which suggests that it has no role in treating the acidemia that occurs during CPR to increase the vasopressor response to epinephrine. However, one limitation of that study was that some 
Table 2. Mean Pressure ( $\mathrm{mm} \mathrm{Hg}$ ) During CPR (n=6)

\begin{tabular}{lcccccc}
\hline & & & \multicolumn{4}{c}{ Minutes after Epinephrine Administration } \\
\cline { 4 - 7 } & INF & $\mathrm{E}$ & 1 & \multicolumn{2}{c}{3} & 4 \\
\hline Bicarbonate & & & & & & \\
$\quad$ AOS & $48 \pm 14.3$ & $48 \pm 14.7$ & $60.5 \pm 18.3$ & $58.1 \pm 17.3$ & $54.7 \pm 17.6$ & $51.9 \pm 13.6$ \\
AOD & $20.7 \pm 8.7$ & $19.3 \pm 10.5$ & $32.3 \pm 13.5$ & $30.3 \pm 12.8$ & $25.6 \pm 12.9$ & $24.4 \pm 13.2$ \\
CPP & $18.4 \pm 8.1$ & $16.5 \pm 8.8$ & $28.9 \pm 12.8$ & $27.3 \pm 11.4$ & $22.9 \pm 11.4$ & $22.1 \pm 12.0$ \\
Normal saline & & & & & & \\
AOS & $42.3 \pm 7.6$ & $45 \pm 9.9$ & $73.4 \pm 15.9$ & $70.3 \pm 17.7$ & $68.0 \pm 15.6$ & $65.6 \pm 12.9$ \\
AOD & $14.0 \pm 7.0$ & $17.5 \pm 9.5$ & $40.4 \pm 21.6$ & $37.1 \pm 22.1$ & $34.6 \pm 20$ & $32.4 \pm 19.1$ \\
CPP & $11.2 \pm 8.5$ & $12.7 \pm 10.8$ & $32.7 \pm 21.4$ & $29.6 \pm 21.3$ & $28.2 \pm 19.8$ & $25.9 \pm 19$ \\
\hline
\end{tabular}

$\mathrm{INF}=$ start of either sodium bicarbonate or normal saline infusion; $\mathrm{E}=$ at the time of epinephrine administration; $\mathrm{AOS}=$ aortic systolic pressure; $\mathrm{AOD}=$ aortic diastolic pressure; $\mathrm{CPP}=$ coronary perfusion pressure.

animals receiving sodium bicarbonate still had arterial acidemia $(\mathrm{pH}<7.4)$, which may have potentially altered sodium bicarbonate's influence on epinephrine's vasopressor effect. In the current study we administered high-dose sodium bicarbonate $3 \mathrm{mEq} / \mathrm{kg}$ to avoid this limitation and to determine the effects of extreme alkalemia on the vasopressor response to epinephrine. If sodium bicarbonate is to affect the vasopressor effect of epinephrine, it should be apparent at the $\mathrm{pH}$ achieved in this study. However, as demonstrated, even extreme alkalemia does not improve epinephrine's ability to increase pressure. This was apparent throughout the study, including at the time of epinephrine's peak effect that corresponded to the time of the highest measured $\mathrm{pH}$.

The results of this study were similar to those

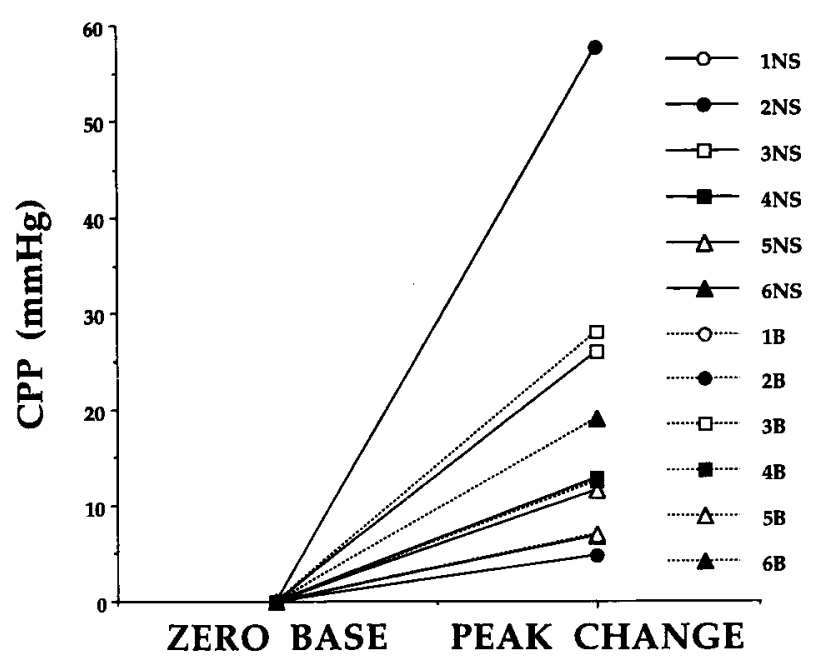

Figure 1. Peak change in coronary perfusion pressure (CPP) for each animal. NS = normal saline group; $\mathrm{B}=$ sodium bicarbonate group; zero base = zero baseline. of our previous study evaluating standard-dose sodium bicarbonate. In addition, they are similar to those of a study in swine that evaluated sodium bicarbonate $3 \mathrm{mEq} / \mathrm{kg}$ and epinephrine $0.04 \mathrm{mg} / \mathrm{kg}{ }^{6}$ However, some important differences exist between the studies, including the type of CPR performed (open- vs closedchest), and baseline coronary perfusion pressure, which was approximately twice as high as that in our study (16.5 vs $30 \mathrm{~mm} \mathrm{Hg}$ ). Of the two studies, ours probably most represents the clinical setting secondary to the type of model used (closed chest) and low coronary perfusion pressures. Despite the differences, both studies have applicable findings and important similar results.

The dose of sodium bicarbonate $3 \mathrm{mEq} / \mathrm{kg}$ was selected to ensure that all animals developed significant arterial alkalemia and was based on previous reports. ${ }^{6-8}$ Sodium bicarbonate was infused over 2 minutes to minimize the vasodilating effects of a hyperosmolar solution, which may decrease coronary perfusion pressure. In a previous study in which a similar sodium bicarbonate dose was infused over 1 minute, the mean decrease in coronary perfusion pressure was from 15 to $9 \mathrm{~mm} \mathrm{Hg.}{ }^{8}$ In our study the coronary perfusion pressure decreased by a mean of $1.9 \pm 4 \mathrm{~mm} \mathrm{Hg}$ after sodium bicarbonate and increased by a mean of $3 \pm 5 \mathrm{~mm} \mathrm{Hg}$ after normal saline $(p=0.12)$. Therefore, by administering sodium bicarbonate over 2 minutes we avoided significant transient decreases in coronary perfusion pressure that could have biased the results.

Our findings have to be interpreted with caution since the model represents a short period of ventricular fibrillation and CPR. It is not known what might occur with longer periods of ventricular fibrillation and $C P R$ at similar doses 
of sodium bicarbonate and epinephrine. Furthermore, our results may have been different if the arterial acidemia induced in the normal saline group had been more severe (i.e., $\mathrm{pH}<$ 7.0). However, clinically this degree of acidemia is rarely seen due to arterial hypocarbia induced by aggressive ventilation. Finally, the previous episode of ventricular fibrillation and CPR may have altered the effects of epinephrine and sodium bicarbonate by some unknown mechanism. Our findings of increased coronary perfusion pressure after epinephrine administration and increased arterial $\mathrm{pH}$ after sodium bicarbonate administration are consistent with the effects of these drugs in animals undergoing only one episode of ventricular fibrillation and $C P R$.

In conclusion, the significant arterial alkalemia induced by high-dose sodium bicarbonate 3 $\mathrm{mEq} / \mathrm{kg}$ had no significant effect on the vasopressor response to epinephrine compared with normal saline in this closed-chest model of ventricular fibrillation and CPR in dogs. These results, together with findings from other studies, suggest that a limited rationale exists for administering sodium bicarbonate during ventricular fibrillation and CPR to treat arterial acidemia in an attempt to improve the vasopressor response to epinephrine.

\section{References}

1. Korstanje $C$, Mathy MJ, van Charldorp $K$, de Jonge $A$, van Zwieten PA. Influence of respiratory acidosis or alkalosis on pressor responses mediated by alpha-1 and alpha-2 adrenoceptors in pithed normotensive rats. Naunyn Schmiedebergs Arch Pharmacol 1985;330:187-92.

2. deHurtado MCC, Argel MI, Cingolani HE. Influence of acidbase alterations on myocardial sensitivity to catecholamines. Naunyn Schmiedebergs Arch Pharmacol 1981;317:219-24.

3. Grant TL, McGrath JC, O'Brien JW. The influence of blood gases on alpha-1 and alpha- 2 adrenoceptor-mediated pressor response in the pithed rat. Br J Pharmacol 1985;86:69-77.

4. Wildenthal K, Mierzwiak DS, Myers RW, Mitchell JH. Effects of acute lactic acidosis on left ventricular performance. Am J Physiol 1968;214:1352-9.

5. Bleske BE, Rice TL, Warren EW, de las Alas VR, Tait AR, Knight PR. The effect of sodium bicarbonate administration on the vasopressor effect of high-dose epinephrine during cardiopulmonary resuscitation in swine. Am J Emerg Med 1993;11:439-43

6. Federiuk CS, Sanders AB, Kern KB, Nelson J, Ewy GA. The effect of bicarbonate on resuscitation from cardiac arrest. Ann Emerg Med 1991;20:1173-7.

7. Gazmuri RJ, von Planta M, Weil MH, Rackow EC. Cardiac effects of carbon dioxide-consuming and carbon dioxidegenerating buffers during cardiopulmonary resuscitation. J Am Coll Cardiol 1990;15:482-90.

8. Kette F, Weil MH, Gazmuri RJ. Buffer solutions may compromise cardiac resuscitation by reducing coronary perfusion pressure. JAMA 1991;266:2121-6. 